\title{
DApp-Didáctica interactiva basada en el uso de los Dispositivos y Apps Propuesta metodológica para la representación del proyecto de arquitectura
} \author{
architectural project \\ - Adriana Edith Granero \\ Universidad de Belgrano, Buenos Aires, Argentina Universidad de Belgrano, Buenos Aires, Argentina \\ adriana.granero@comunidad.ub.edu.ar \\ liliana.bonvecchi@ub.edu.ar \\ - Mónica Inés Fernández \\ Universidad de Belgrano, Buenos Aires, Argentina \\ Mabel Clara Brignone \\ Universidad de Belgrano, Buenos Aires, Argentina \\ monica.fernandez@ub.edu.ar \\ mabel.brignone@comunidad.ub.edu.ar
}

DApp - Interactive teaching methodological proposal based on the use of the devices and Apps for the representation of the

\begin{abstract}
A common problem we share teachers today is the presence of smartphones and tablets in the classroom; these electronic devices generate distractions of its use for communication in social networks, games, and visits to Internet sites. These elements are at all times available to the student, which is why we raised the need to adapt some learning processes with their interaction. The intention is to demonstrate that these practices allow for the introduction of theoretical concepts and fundamentals related academic imagination, the design process and representation of the architectural project. The proposal linked with an increase of the professional needs that the architect faces, after their training, professional skills to perform in today's job market.
\end{abstract}

Keywords: Educación, Interacción, Aplicaciones, Teléfono Inteligente, Smart-TV

\section{Introducción}

Un problema común que compartimos los profesores en la actualidad, es la presencia de teléfonos inteligentes y tabletas en el aula; estos dispositivos electrónicos con conectividad inalámbrica generan, en más de una ocasión, distracciones vinculadas a su uso para la comunicación en redes sociales, juegos, visitas a páginas de Internet, etc. Estos elementos se encuentran en todo momento al alcance del alumno, razón por la cual planteamos la necesidad de adaptar algunos procesos didácticos en interacción con los mismos y así generar nuevas alternativas de uso y de prácticas.

Este trabajo expone asimismo la exploración de una estrategia didáctica de vinculación emocional. Para realizar la experiencia, hemos propuesto la interacción con distintos dispositivos de comunicación y con diversas aplicaciones. La intención es demostrar que estas prácticas permiten la introducción de conceptos y fundamentos teórico-académicos relacionados con la imaginación, el proceso de diseño y las cualidades expresivas aplicadas en la representación del proyecto arquitectónico.

Para el testeo, los dispositivos utilizados fueron teléfonos inteligentes, tabletas, y Smart-TV.

Asimismo, la propuesta se enlaza con el avance de las necesidades profesionales a las que se enfrenta el arquitecto, una vez finalizada su formación. Tales desafíos evolutivos se relacionan con el uso de las nuevas tecnologías, el trabajo en equipo a distancia, así como la capacidad de innovar y de emprender nuevos desafíos. Estos nuevos encuentros tecnológicos, someten el ejercicio de la profesión a transformaciones importantes que hacen fundamental anticipar y adaptar la oferta formativa, con la finalidad de formar futuros profesionales en las competencias necesarias para desempeñarse en el mercado laboral actual.

\section{Procesos Metodológicos}

El procedimiento empleado en esta investigación inicia con una estrategia general de revisión teórica, un análisis de los dispositivos y Apps disponibles, y la incorporación de ensayos secuenciados con los estudiantes. La simultaneidad de la información a través de la posibilidad de visualizar varias ventanas operativas, como también el recurso de edición y procesamiento digital, que permite manipular las imágenes, combinarlas y trasladarlas a diferentes soportes, las capturas generadas desde una aplicación scanner o registro fotográfico, hace que un boceto o un croquis hecho a mano pueda participar de las mismas operaciones que una fotografía digital obtenida de una maqueta táctil. A la vez que dispositivos Apps permiten generar modelos concretos y físicos, reconstruyendo la apariencia "transparente e inmaterial del espacio digital en espacios físicos y hápticos posibles de ser integrados a la realidad sensible, participando del espacio imaginario y real. 
Antes de describir los procedimientos metodológicos, cabe aclarar que en la propuesta de apropiación de conocimientos a través de aplicaciones móviles gratuitas, el modelo didáctico utilizado por el alumno (transparente para sí mismo) es el auto-constructivismo. Este mecanismo se asocia con el autoconstructivismo-lúdico, aprendido en las etapas iniciales de desarrollo, consecuencia del contacto directo con los videos juego o juegos en red (BAÑARES, 2008).

Para que surja la apropiación de conocimientos se hace necesario plantear situaciones que estimulen la participación y el protagonismo. Por este motivo y para provocar estas acciones, se hace necesaria una metodología didáctica en la que se haga uso de los dispositivos y Apps, un sistema que favorezca la interacción entre lo imaginario y lo real.

La propuesta metodológica involucra la actividad lúdica, tiene la finalidad de generar espacios de aprendizaje basados en el interaccionismo simbólico (DUSSEL, 2010), forma parte del paradigma interpretativo y permite llegar al conocimiento de manera significativa a través de atajos holísticos (ESNAOLA, 2006). La estructura de la ejercitación programada se fundamenta en metas $u$ objetivos sobre temáticos específicos, en la comunicación de reglas y en los instrumentos que permitirán llegar a esas metas. (BERTERO, 2009)

Las etapas a cumplir son las siguientes: en primera instancia, presentar una meta u objetivo de interés para el alumno que cumpla, además, con los requisitos necesarios para transferir conocimientos; el paso siguiente es la creación de un modelo real, a escala, de elementos geométricos espaciales (sólidos platónicos) con técnicas tradicionales (cartón, madera, o materiales similares), relacionados por encastre y recreando un límite que encierra el espacio arquitectónico; el tercer paso es la captura fotográfica de la maqueta arquitectónica inicial, a través del Smartphone. Concluidas estas acciones, el paso siguiente es el tratamiento, con Apps gratuitas, de las imágenes obtenidas. La búsqueda es estimulada por el docente, quien pone un ejemplo en conocimiento de los alumnos, mostrando las posibilidades de aplicación e intervención. Luego se estimula a investigar y catalogar las distintas Apps en un trabajo colaborativo, para elaborar posteriormente un informe. El resultado de esta ejercitación formará parte de una comunicación gráfica visual, una síntesis del proceso de aprendizaje en técnicas de representación del proyecto arquitectónico.

La aplicación de las Apps persigue la integración de las actividades realizadas en el campo teórico-práctico, físico-real y digital, de la representación conceptual de la arquitectura.

Repensar desde el ámbito técnico el uso de los dispositivos, tanto teléfonos inteligentes como tabletas, planteando la creación de un Laboratorio Apps Intermedial, se propone desde lo didáctico, como un lugar donde dispositivos y Apps conviven y se potencian para desarrollar nuevas técnicas didácticas $\mathrm{y}$, combinaciones intermediales, sino también como espacios interdisciplinares, que permiten acceder a lugares poco explorados del campo técnico-programático tratando de encontrar como decía (MACHADO, 2006) de qué manera invertir las funciones técnicas en función a la productividad ya programada. La propuesta de un uso integrado de Apps aplicadas a enseñanza sugiere en proyectiva su catalogación para que se constituyan en herramientas dinámicas para su uso en arquitectura y diseño. De esta manera se generan agrupamientos colaborativos de diferentes Apps, que pueden ser utilizados en las diferentes temáticas y conceptualizaciones.

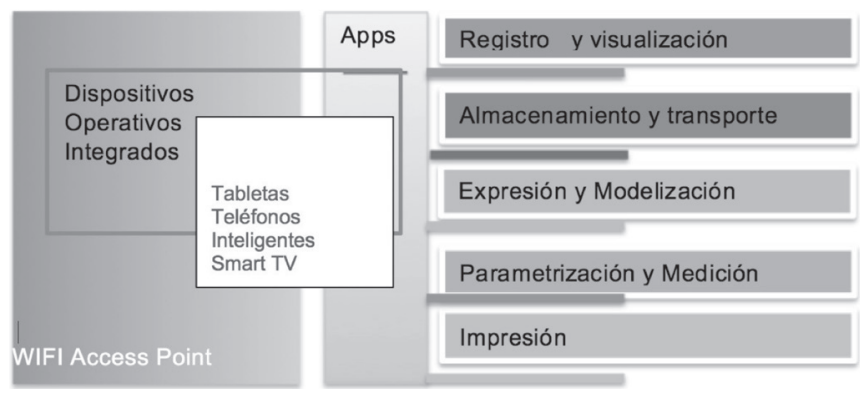

Figura 1: Esquema catalogación de Apps en relación a la conceptualización temática y operativa.

La actividad se plantea como apoyo de procesos creativos y su fundamento se relaciona con la capacidad que posee el alumno de interactuar, realizar varias actividades al mismo tiempo así como catalogar (SCHOOLNET, 2014) y procesar el resultado de la interacción (PPD-Procesamiento Paralelo Distribuido). También se toma en cuenta la facilidad que posee el estudiante para relacionar y asociar conceptos (MA - Memoria Asociativa) (BASBAUM, 2005), así como su habilidad para comparar y analizar información definiendo igualdad o diferencia (AP-Apareamiento de Patrones) (SCHÖN, 1998). Otro factor a considerar es la Auto-organización (LION C. ,2006), como consecuencia de la aplicación de un sistema de procesamiento en paralelo (VAINA, 2010), adquirida en etapas tempranas a partir del uso de videojuegos. Su finalidad es ser sustento de la resolución de problemas presentados en el desarrollo del proyecto. La propuesta establece unidades de producción de conocimiento, con trabajo individual y cooperativo.

En este proceso, el alumno llegará a conocer el espacio y la representación en el plano digital desde su teléfono inteligente, podrá reelaborar la comunicación entre pares y con el docente, compartiendo el trabajo desde el mismo al Smart TV, ubicado en el taller donde se dicta la asignatura (PÉREZ LINDO, 2012). Esta actividad generará una reflexión comunitaria guiada por el docente. El propósito de la experiencia es descubrir el procedimiento de apropiación de saberes (RENEÉ CANDIA, 2006). La introspección en el reconocimiento de una tarea táctica, permitirá el tratamiento de la información en forma distribuida y coordinada, a través de una actividad natural, en la que puede operar y adquirir destrezas con herramientas y aplicaciones a su alcance. (MONEDERO MOYA, 2007) 
En esta didáctica interactiva, orientada la aprehensión de técnicas de representación para el proyecto arquitectónico, se integran una serie de pasos ordenados lógicamente y sistematizados. La propuesta deviene en un plan de actividades que tiene origen en el programa de la asignatura, ejecutado por los estudiantes para llegar a un fin, la representación y comunicación de la Arquitectura (BERTERO, 2009).

Se prevé únicamente una clase de presentación inicial, workshops, sin clases especiales ni clases teóricas. En este contexto, el docente desarrolla temáticas, suministra información a partir de las demandas de cada trabajo particular (aprendizaje justo a tiempo) (ZAPATA-ROS, 2012), plantea el trabajo dentro del taller como aplicación del método de pautas y guía al estudiante para llegar a los resultados esperados.

Se sugiere que, con el empleo de acciones lúdicas instrumentales y por auto-mayéutica se estimule el desarrollo de habilidades técnico-cognitivas (SCIALABBA., 2012) vinculadas a la representación de la arquitectura. Un manejo razonado de la información vinculada con la técnica de representación digital y el desarrollo de pensamiento creativo, se relaciona con la pre-figuración y con el arte, en el ámbito de la enseñanza de la carrera de Arquitectura.

\section{Resultados}

La investigación se encuentra en curso, actualmente en la segunda etapa, los ensayos de la primer etapa muestran resultados parciales y son los siguientes: el $75 \%$ de los participantes pudo realizar la experiencia, el 85\% dejó de utilizar su dispositivos para comunicarse en las redes sociales mientras estaban trabajando con la aplicación durante la clase presencial en el taller, el 100\% de los estudiantes manifestó que no tenía conocimiento de las Apps y su utilidad para la actividad relacionada a su aprendizaje y a su actividad profesional futura. La experiencia se realizó con alumnos de $1^{\circ}$ año de la carrera de Arquitectura en la Universidad de Belgrano, en la materia Forma y Comunicación, tuvo un desarrolló de 3 clases; las mismas fueron completadas en distintas etapas, para la internalización de distintas conceptualizaciones temáticas y con distintas aplicaciones.

Así mismo se modificaron los elementos utilizados para la evaluación y la cuantificación de las tareas asignadas, esta labor se llevó a cabo a modo de rúbrica con una tabla de jerarquización en cuanto a la aplicación y capitalización de
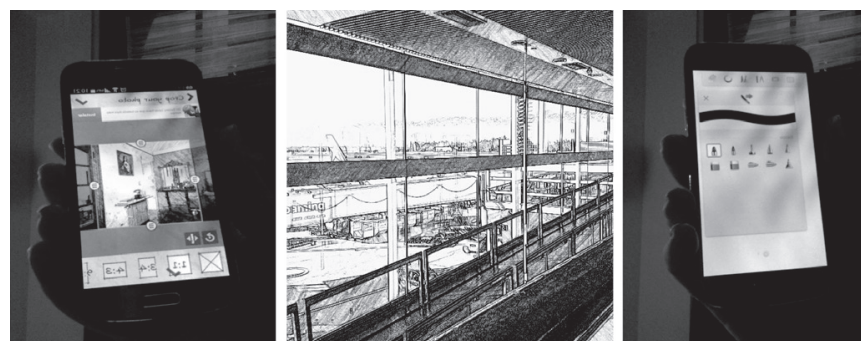

Figura 2: Usos y prácticas. los conocimientos adquiridos, tanto en el manejo operativo del dispositivo App como en el resultado generado a partir del uso del mismo. Es de destacar que el uso de éstas Apps como herramientas didácticas, permiten también elaborar mayor cantidad alternativas y generar más elementos de reconocimiento para ser visualizadas y corregidas en simultáneo con el docente, y así perfeccionar las mismas operatorias generando nuevos catalogaciones y alternativas sobre una misma propuesta.

El propósito del Proyecto Metodológico Didáctico DApp es orientar al alumno en la auto-construcción de su conocimiento, en referencia a la representación de la arquitectura, con utilización de dispositivos como teléfono inteligente, Tablet, instrumentos tecnológicos que están permanentemente en poder del alumno, de uso común para otras actividades vinculadas con su vida social y con situaciones placenteras, que se presentan, en esta experiencia, integrados a la enseñanza. La utilización del Smart-TV se manifiesta, además, como un medio de interacción con sus pares y profesores, quienes se integran conformando un circuito dinámico y participativo sobre las propuestas proyectuales generadas.

Desde la ubicuidad como otra característica significativa de estos dispositivos se construye un aprendizaje que se suele definir como el que se produce en cualquier lugar y momento; así la tecnología ubicua (informática cercana a la persona, por ejemplo, un móvil) potencia considerablemente este tipo de metodología de aprendizaje (LION \& M., 2012). La formación ubicua integra el aprendizaje y la tecnología ubicua dentro de una estrategia formativa y uno de sus frutos más conocidos es el m-learning (utilización de dispositivos móviles para el aprendizaje).

Como ya es tradicional, cada vez que surge una nueva tecnología, lo primero que se suele hacer es traspasar los contenidos a un formato que pueda ser tratado por esa tecnología. Evidentemente, si los contenidos y actividades formativas son accesibles a través de tecnologías ubicuas y estas siempre nos acompañan, se facilita considerablemente el acceso a los recursos formativos y colaborativos posibilitando también la integración y el intercambio grupal como facilitadores en la construcción del conocimiento, en referencia a la representación y comunicación de la Arquitectura. La utilización del Smart-TV en el espacio taller se constituye como un medio de interacción con sus pares y profesores, colaborando en una didáctica ágil e interactiva .
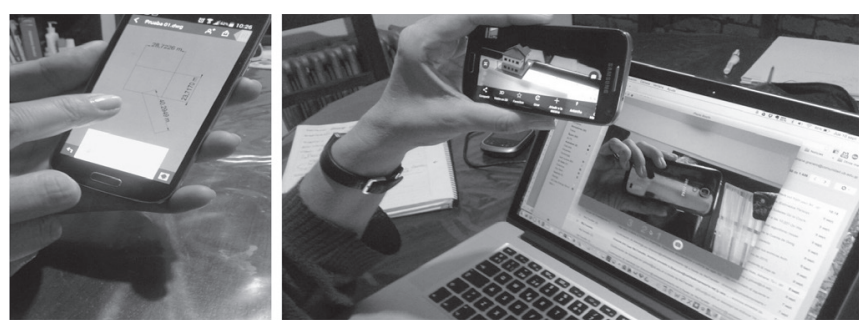

Figura 3: Interacción de dispositivos. 


\section{Discusión}

El impacto que esta propuesta pretende generar, es un cambio de paradigma didáctico, al integrar dispositivos digitales a la enseñanza-aprendizaje con la intención de convertir el modelo en uno que relacione activa y emocionalmente lo que se aprende. De esta manera, una construcción aparentemente autónoma del conocimiento, realizada a partir de la auto-reflexión y guiada por el docente, provocada por el empleo de instrumentos tecnológicos de uso corriente, actúa como mediadora para la comunicación y la representación de la Arquitectura. El Smartphone, Tablet o Smart Tv, funcionan como vehículos de pensamiento, como productores de tecno-conocimiento, en un proceso de clivaje comunicativo en la acción de ejemplificar y reflexionar.

La intervención del docente alude a la forma, a la función, a la tecnología, a la incorporación de referencias, al contexto y al valor significativo de la obra. Si bien alguno de los problemas que plantea la propuesta, es la necesidad de formación de los docentes, es evidente que para transferir el concepto de interacción e integración, es necesario estar en conocimiento de los detalles del proceso y del funcionamiento de los dispositivos. Su capacitación esta en relación con los cambios epocales que las tecnologías generan en el espacio cotidiano de su uso y sus extensiones.Dentro de este proceso es posible determinar tres aspectos que se encadenan como una sucesión de intervenciones: los contenidos, el proceso productivo y el producto final.

Así como la integración espacial de máquinas y dispositivos constituyen un espacio real de capacitación interacción y producción simultánea, en el que el manejo y la operatoria del dispositivo hace visible la acción humana que se proyecta en la producción y en los resultados finales.

La integración de dinámicas de modelización y procesamientos digitales funcionan como una alquimia práctica, como plantea Zielinski (2007), donde señales analógicas y códigos digitales funcionan como un intento de hacer visibles y sensibles las cualidades de la apariencia física del objeto, que fueron generadas en una realidad virtual y luego procesadas desde Apps específicas a través de operaciones que se complementan con la reconstrucción y armado, donde el trabajo manual sobre las maquetas de objetos diseñados completan su realización.

La posibilidad de concebir hoy un Taller Expandido e Integrado como un espacio proyectual de diseño, fabricación y expresión analógico-digital simultánea, junto a la representacion de espacios virtuales en 3D recrean por momentos antiguos espacios de trabajo del siglo XVII al XIX, donde convivían e interactuaban en la construcción de un conocimiento, donde cuerpo y mente participaban activamente de la construcción desde la herramienta y las máquinas como facilitadora del hacer y el pensar.

Este espacio de alquimias y prácticas es el espacio del artesano y el investigador del renacimiento, el cual se resignifica hoy, a través del alumno quien como funcionario y operador construye desde su cuerpo y mente esta integración y proyecta su saber al hacer, aquí es donde surge la figura de un perfil de alumno como artesano digital, que comienza a autoformarse desde la integración de dominios y saberes que desde analogización digital proponen nuevas interacciones con las dinámicas de los dispositivos.
DISPOSITIVOS Y APPS

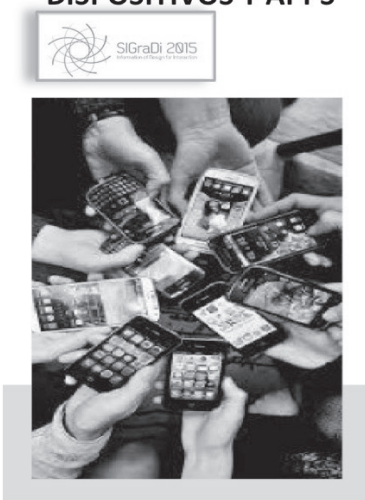

Propuesta Interactiva didáctica basada en el uso de los Dispositivos y Apps para la representación del proyecto de Arquitectura

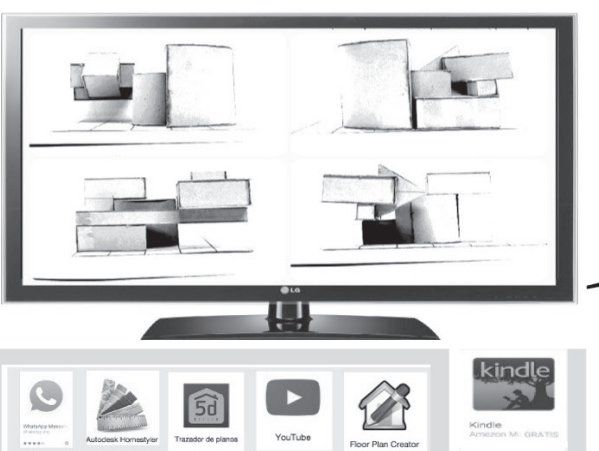

La utilidad de los Dispositivos y la Apps como sistema que favorece la interacción entre lo imaginario y lo real
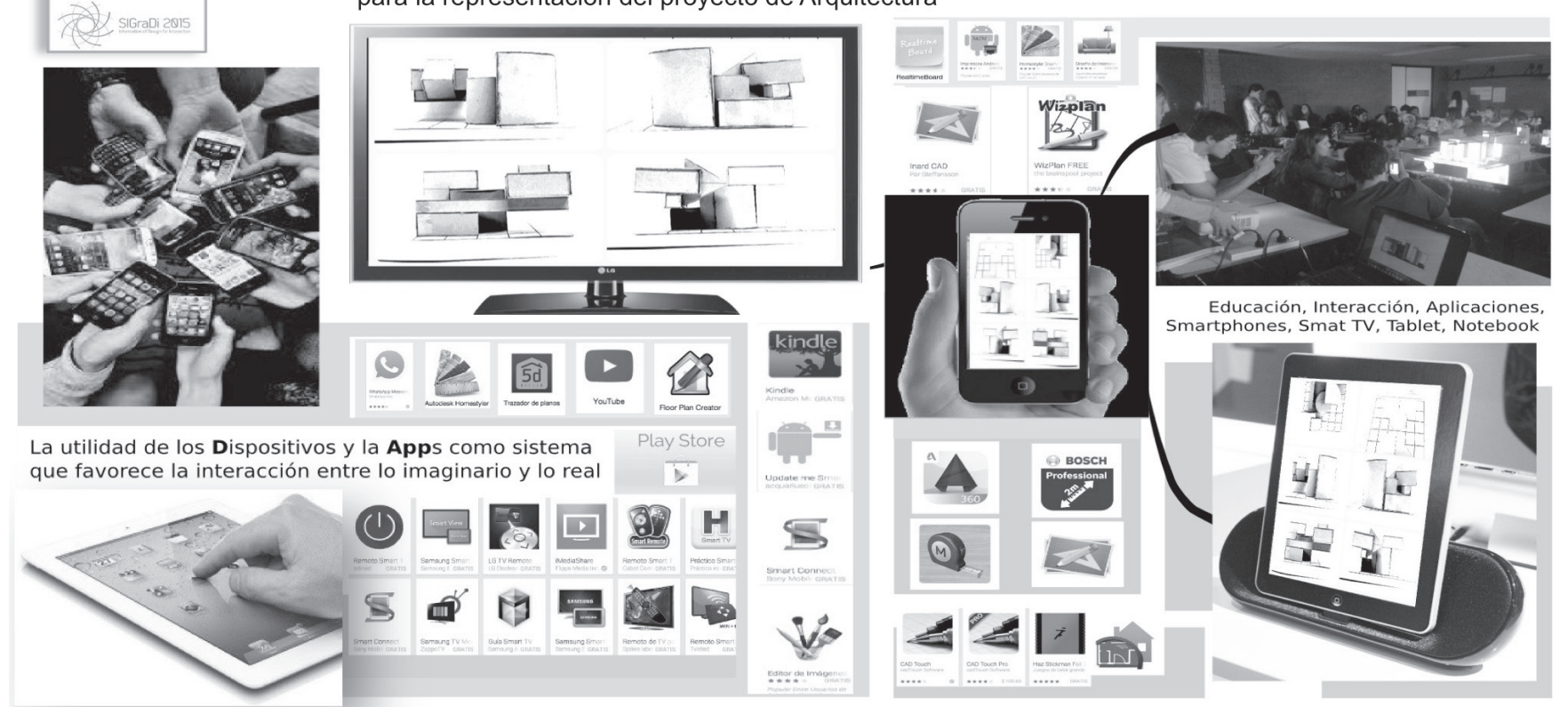

Figura 4: Proyecto metodológico didáctico DApp. 


\section{Referencias}

Arnheim, R. (2008). Arte y percepción visual. Psicología del ojo del creador (Nueva Versión). [trans.] M.L. BALSEIRO. 4. Madrid: Alianza Forma, 2008. 978-84-206-7874-0.

(1985). El pensamiento visual. [trans.] R. MASERA. $1^{\circ}$. Buenos Aires: Paidós, 1985. 85-7509-377-9.

Bañares, D. and al, et. (2008). El juego como estrategia didáctica: Claves para la innovación educativa. $1^{\circ}$. Caracas : GRAÓ de IRIF SL, 2008. 978-84-7827-633-2.

Basbaum, S. (2005). O primado da percepÇao e suas consecuencias no ambiente mediatico. Tesis de Doctorado en Comunicación y Semiótica. Sao Paulo, Sao Paulo, Brasil: PUC/ SP, 11, 2005.

Bertero, C. (2009). La enseñanza de la arquitectura: entre lo dibujado y lo desdibujado. $1^{\circ}$. Santa Fe: Universidad Nacional del Litoral, 2009. 978-987-657-065-7.

Dussel, I. (2010). VI Foro Latinoamericano de Educación; Educación y nuevas tecnologías: los desafíos pedagógicos ante el mundo digital. $1^{\circ}$. Buenos Aires: Santillana, 2010. 978-95046-2252-9.

EDUTEKA. 2014(Online) 5.14,2015 http://www.eduteka. org/pdfdir/reportehorizonte-K12-2014.pdf EUROPEAN SCHOOLNET.2014.InnovaveToolsintheclasroom:Implications for learning styles and gender. [Online] 4 15, 2015. http:// www.eun.org/c/document_library/get_file?uuid $=c 47531 \mathrm{fc}-$ 5d3f-41ab-86c9-f19cf8858a55\&groupId=43887

Esnaola, G. (2006). Claves Culturales En La Construccion Del Conocimiento ¿Qué enseñan los videojuegos? $\left(1^{\circ}\right.$ ed.). Buenos Aires, Argentina: Alfagrama.

Gardner, H. (2003). La inteligencia reformulada: las inteligencias múltipes en el siglo XXI. [trans.] Genís Sánchez Barberán. 1․ Buenos Aires: Paidós, 2003. 978-84-493-1029-4. Lion, C. (2006). Imaginar con tecnologías. Buenos Aires, Argentina: La Crujía.

Lion, Carina (2012) "Pensar en red: metáforas y escenarios". En: M. Naradowski y A. Scialabba. Comps. ¿Cómo serán? El futuro de la escuela y las nuevas tecnologías. Buenos Aires. Prometeo.

Machado,Arlindo, (2006)Convergencia y divergencia de los medios" en: Miradas,EICTV,LaHabana http://www.eictv. co.cu/miradas/index.php
Narodowski and A. (Compiladores) SCIALABBA.¿Cómo serán?: el futuro de la escuela y las nuevas tecnologías. $1^{\circ}$. Buenos Aires: Prometeo Libros, 2012. p. 222. 978-987-574-5582. pp. 29-45.

Litwin, E. (Comp.). (2005). Tecnologías educativas en tiempo de internet. Buenos Aires: Amorrortu, 2005.

Monedero Moya, J. (2007). El diseño de los materiales educativos ante un nuevo reto en la enseñanza universitaria: el Espacio Europeo de Educación Superior (EEES). 1, 2007, Interuniversitaria de Formación del Profesorado, 21(1), Vol. 21, pp. 51-68.

Monedero, M. (2007) Interuniversitaria de Formación del Profesorado, Vol.21, pág. 51-68. 0213-8464

Padron Nápoles, C. (2009). Desarrollo de materiales didácticos desde una perspetiva basada en modelos. Tesis Doctoral. Luganes, España: s.n., 2009.

Pérez Lindo, A. (2012). Capacidad para enseñar a aprender. En A. pérez lindo, Competencias docentes para el siglo XXI (págs. 81-88). Buenos Aires, Argentina: Tinta Fresca.

Reneé candia, M. (2006). Jerome Bruner - Aprendizaje por descubrimiento. La organización de situaciones de enseñanza: unidades didácticas y proyectos, articulación con talleres, actividades de rutina. Buenos Aires: Centro de Publicaciones Educativas y Material Didáctico, 2006, pp. 52-53.

Schön, D. (1998). El profesional reflexivo: Como piensan los profesionales cuando actúan. Buenos Aires: Paidós, 1998. 84493-0556-X.

Trebilcock, M. (2009). Proceso de Diseño Integrado: nuevos paradigmas en arquitectura sustentable. Obtenido de Redalyc: http://redalyc.uaemex.mx/src/inicio/ArtPdfRed. jsp?iCve $=193614470001$

Vaina, L. (2010). Vision: A Compattional Investigation Into The Human Representatios and Processig of Visual Infotmation/ David Marr. Cambridge, Massachuset: The MIT Press, 2010.

Zapata-Ros, M.l. (2012). Teorías y Modelos sobre el aprendizaje en entornos conectados y ubicuos. Base para un nuevo modelo teórico a partir de una visión crítica del "conectivismo". Alcalá: E-Lis Repository, 2012. pp. 14-63.

Zielinski, S. (2007). Siegfried Zielinski: Genealogías, Visión escuchay, comunicación. Bogota /Colombia: UNIANDES -Universidad de los Andes. 